\title{
EFICACIA EN LA IMPLEMENTACIÓN DE PROGRAMAS LINGÜÍSTICOS PARA LA MEJORA DE LAS HABILIDADES COMUNICATIVAS EN PERSONAS CON DEMENCIA
}

\author{
Gema Regatos Corchete \\ Profesora Asociada en Universidad de Pontificia de Salamanca. \\ Facultad de Ciencias de la Salud. \\ Doctoranda en Ciencias Humanas y Sociales. Universidad Pontificia de Salamanca. gregatosco@upsa.es \\ Cristina Gómez García \\ Profesora Asociada en Universidad de Salamanca. Facultad de Psicología. \\ Doctoranda en Ciencias Humanas y Sociales. Universidad Pontificia de Salamanca. \\ cristinagomezgarcia@usal.es \\ Ma Cruz Pérez Lancho \\ Profesora Encargada de Cátedra. Facultad de Ciencias de la Salud. \\ Universidad Pontificia de Salamanca mcperezla@upsa.es
}

Recepción Artículo: 27 octubre 2021 Admisión Evaluación: 27 octubre 2021 Informe Evaluador 1: 28 octubre 2021 Informe Evaluador 2: 29 octubre 2021

Aprobación Publicación: 30 octubre 2021

\begin{abstract}
RESUMEN
Introducción: la estimulación cognitiva predominante en población con deterioro cognitivo se centra en preservar las competencias requeridas para la realización de actividades de la vida diaria que logren mantener la vida autónoma de los pacientes el mayor tiempo posible. El progresivo declive que sufren las personas con demencia afecta de manera significativa a todas las áreas de la persona, incluyendo su capacidad para comunicarse, lo que provoca un gran sentimiento de dependencia y discapacidad. El diseño de programas de estimulación cognoscitiva y del lenguaje apuesta por la promoción del mantenimiento y la compensación de habilidades para la interacción comunicativa efectiva de los pacientes con demencia con su entorno. Objetivo: análisis exploratorio de resultados obtenidos mediante la implementación de programas de estimulación y mantenimiento comunicativo en personas con deterioro cognitivo. Se pretende identificar cambios significativos en pruebas de nominación, evocación y secuencialidad y conocer si dichos diseños ofrecen a los pacientes la posibilidad de mantener y estabilizar múltiples funciones superiores. Metodología: realizamos una revisión bibliográfica sistemática de los 12 últimos años que recoge y sintetiza los principales trabajos cuasiexperimentales en lengua inglesa y castellana. Resultados: evidencia del fortalecimiento de habilidades cognitivas preservadas, así como estimulación de destrezas comunicativas a pesar de la progresión de la enfermedad. Conclusiones: se destaca la importancia de considerar el tipo de deterioro cognitivo y estadio en el que se encuentra la enfermedad para adecuar las actividades según las necesidades de cada persona, utilizando sus fortalezas y reforzando un conjunto de estrategias de comunicación que resultan ampliamente beneficiosas.
\end{abstract}


Palabras clave: demencia; enfermedad de Alzheimer; afasia; tratamiento no farmacológico; rehabilitación; recuperación de léxico; trastornos de la comunicación; terapia cognitiva

\begin{abstract}
Effectiveness in the implementation of language programs for the improvement of communication skills in people with dementia. Introduction: the predominant cognitive stimulation in the population with cognitive impairment focuses on preserving the skills required to carry out activities of daily living that manage to maintain the autonomous life of patients for as long as possible. The progressive decline suffered by people with dementia significantly affects all areas of the person, including their ability to communicate, causing a great feeling of dependency and disability. The design of cognitive and language stimulation programs is committed to promoting the maintenance and compensation of skills for effective communicative interaction of patients with dementia with their environment. Objective: exploratory analysis of results obtained through the implementation of communication stimulation and maintenance programs in people with cognitive impairment. The aim is to identify significant changes in tests of nomination, evocation and sequencing and to know if these designs offer patients the possibility of maintaining and stabilizing multiple superior functions. Methodology: we carried out a systematic bibliographic review of the last 12 years that collects and synthesizes the main quasiexperimental works in English and Spanish. Results: evidence of the strengthening of preserved cognitive skills, as well as stimulation of communication skills despite the progression of the disease. Conclusions: the importance of considering the type of cognitive deterioration and the stage of the disease is highlighted in order to adapt the activities according to the needs of each person, using their strengths and reinforcing a set of communication strategies that are widely beneficial.
\end{abstract}

Keywords: dementia; Alzheimer disease; aphasia; non-pharmacological treatment; rehabilitation; lexicon retrieval; communication disorders; cognitive therapy

\title{
INTRODUCCIÓN
}

El concepto de demencia engloba una gran cantidad de variables clínicas en la sintomatología que presentan los pacientes. Lo que si es común a todos los patrones de declive es la afectación directa que tiene la pérdida progresiva de autonomía en las diferentes áreas de la vida diaria de una persona enferma.

Considerar el lenguaje como una entidad preservada en el deterioro cognitivo ha sido un grave error durante décadas pasadas. Los mecanismos de producción y comprensión lingüística sufren alteraciones desde el inicio del deterioro cognitivo, destacando los cambios en el lenguaje oral, con la presencia de palabras vacías de contenido, dificultades en evocación, circunloquios y pobre fluidez semántica, entre otras (García y Carro, 2011).

El lenguaje es una de las características esenciales de nuestra naturaleza humana ya que nos apoyamos en él para fortalecer todas las demás dimensiones personales. Comunicación es sinónimo de desarrollo social, de enriquecimiento cognitivo y de organización del pensamiento. Evocamos, demandamos y establecemos diferentes relaciones gracias a los intercambios comunicativos. Somos capaces de expresar emociones, de regular nuestra conducta, de cubrir necesidades y de organizar nuestra vida a través del uso de las palabras. El deterioro lingüístico provoca por tanto, una pérdida de autonomía funcional debido a la falta de comunicación siendo ésta la habilidad básica en la interacción humana (Triviño et al., 2021).

Las principales demencias que cursan precozmente con alteraciones comunicativas son la Enfermedad de Alzheimer, con trastornos derivados de la progresión de los déficits de memoria (Bourgeois y Hickey, 2009) y la afasia progresiva primaria, con diversos cuadros clínicos, que incluyen la alteración amnésica y la dominancia del hemisferio izquierdo a nivel de afectación, con prominentes dificultades lingüísticas (McKhann et al., 2011). Cada cuadro clínico presenta distintos perfiles de deterioro lingüístico. En su propuesta más sencilla, el equipo de Mesulam clasifica las variantes en APP con agramatismo, APP o demencia semántica y APP Iogopénica, añadiendo una cuarta categoría, APP mixta, para los casos que no pueden clasificarse en las otras tres (Mesulam, Rogalski, Wieneke, Hurley, Geula, Bigio, Thompson y Weintraub, 2014). Los aspectos que comparten estas 
variantes con la $\mathrm{EA}$, aunque se diferencien en el sustrato neuroanatómico afectado y las diferentes proteinopatías que provocan el daño neuronal, son las dificultades de recuperación lexical, así como una deficiente comprensión en información abstracta y compleja. Siendo el componente de recuperación lexical el aspecto del lenguaje en los que se centran predominantemente, los programas de entrenamiento comunicativo que se dirigen a personas con demencia en general.

Por otro lado, el deterioro cognitivo va enlazado con pérdida de habilidad comunicativa, motivo por el cual se considera atractivo implementar programas que estimulen dicha habilidad, impidiendo un avance vertiginoso asociado al progresivo deterioro que produce la demencia. La mayoría de las investigaciones analizadas están destinadas a mejorar la recuperación del léxico, logrando resultados positivos que se mantienen durante períodos de tiempo que van desde una semana a seis meses, fortaleciendo la hipótesis de que es posible mejorar y mantener las habilidades comunicativas de los pacientes (Jokel et al., 2014).

La validez e importancia de los programas lingüísticos es defendida por autores como Henry et al. (2013) al apostar por el entrenamiento continuo post tratamiento para favorecer el mantenimiento de la competencia de denominación adquirida durante la aplicación de los programas cognitivos. Llega a considerarse que puede disminuir la progresión del deterioro cognitivo a pesar de que la degeneración de los lóbulos temporales siga su curso (Brambati, 2009; Krueger et al., 2010).

\section{OBJETIVOS}

Este trabajo de revisión bibliográfica selecciona los estudios realizados sobre programas de estimulación cognitiva integral que contengan un abordaje de aspectos relacionados con el lenguaje y la comunicación.

El objetivo principal de la revisión bibliográfica se centra en analizar las evidencias relativas a la eficacia de la implementación de programas lingüísticos en personas con demencia.

El segundo objetivo persigue identificar aquellas variables que pueden ser facilitadoras de una intervención lingüística positiva en el entrenamiento de la recuperación léxica.

\section{METODOLOGÍA}

\section{Materiales}

Se seleccionan 18 estudios publicados durante los doce últimos años en lengua inglesa y castellana sobre programas de intervención no farmacológica cognitiva en pacientes con demencia. De ellos, 6 estudios incluían pacientes con afasia progresiva primaria de vertiente semántica y logopénica,10 a pacientes con demencia semántica y 2 a pacientes con demencia tipo Alzheimer. Todos ellos incluyen en su programa de intervención la estimulación lingüística.

\section{Procedimiento}

Para realizar la selección de los artículos se realizó una búsqueda en bases de datos electrónicas como PubMed, Scopus, Medline, PsycINF0, PsycArticles, Cinahl y Dialnet.

Los descriptores empleados fueron: demencia; enfermedad de Alzheimer; afasia; tratamiento no farmacológico; rehabilitación; recuperación de léxico; trastornos de la comunicación y terapia cognitiva.

Los criterios específicos para la inclusión de estudios en esta revisión consistieron en seleccionar: (a) publicaciones entre 2009 y octubre de 2021, (b) personas diagnosticadas con enfermedad de Alzheimer, demencia semántica, o afasia progresiva primaria, (c) programas cuasiexperimentales de corte cognitivo, (d) intervenciones basadas en la estimulación de la recuperación léxica (recuperación léxica, búsqueda de palabras, anomia, terapia del lenguaje).

Posteriormente, cada estudio fue analizado atendiendo a: (a) las características de los participantes (sexo, edad, diagnóstico, tiempo que había pasado desde la aparición de los primeros síntomas relacionados con dificultades lingüísticas y síntomas principales), 
(b) el diseño de intervención (objetivos, elementos, naturaleza y modalidad de los estímulos, interacción y tipo de facilitación, dosis y temporalización), (c) los resultados obtenidos (a nivel expresivo, comprensivo, de relación directa al entrenamiento y de generalización a otros contextos).

\section{RESULTADOS}

En primer lugar, analizamos el soporte de los programas. Varios autores incorporaron soportes digitales para implementar los programas de entrenamiento. Autores como Croot et al. (2015) realizan una intervención basada en la denominación de imágenes proporcionando representación ortográfica y fonológica a través de software digital, mediante el programa de Mircosoft Office, PowerPoint. Por su parte, Mayberry et al. (2011) apoyaron el entrenamiento de denominación de imágenes junto a la correspondiente palabra escrita, obteniendo una mejora significativa en los ítems trabajados. Otros investigadores como Savage et al. (2014) también utilizaron software de ordenador para proyectar los ítems. Cabe destacar que, además de obtener mejorías en la denominación de los ítems, Senaha et al. (2010) obtuvieron resultados positivos también en la definición de los mismos. La utilización del software MossTalk Words, soporte básico para la presentación de imágenes, fue el elegido por Jokel et al. (2009), para la realización de tareas de denominación.

En segundo lugar abordaremos los componentes semánticos y sintácticos de los programas. Aunque la mayoría de los estudios analizados centraron su investigación en la recuperación y producción léxica de forma aislada, autores como Jokel, Cupit, Rochon y Leonard (2009); Robinson et al. (2009); Jokel et al. (2010); Jokel y Anderson (2012); Savage et al. (2014) y Suárez-González et al.(2015), investigaron acerca de la generalización del tratamiento a nivel oracional. De nuevo, se obtienen resultados prometedores en la dimensión sintáctica y semántica del lenguaje. En referencia a la generalización de aprendizajes al discurso, éste fue analizado por autores como Heredia et al. (2009); Beeson et al. (2011); Croot et al. (2015) y Beales et al. (2016), afirmando la existencia de una mayor riqueza léxica de uso diario y perfeccionamiento en la dicción de los pacientes sometidos al programa de estimulación lingüística.

Por último, analizamos los niveles de mantenimiento y compensación de habilidades pragmáticas. En 2012 , Riaño y Moreno implementan un programa de estimulación cognoscitivo-comunicativa, marcándose como objetivo el mantenimiento y compensación de habilidades que permitiesen realizar una interacción comunicativa con el entorno. Las actividades trabajadas se centraron en el desarrollo de la interacción comunicativa espontánea, denominación, lectura y escritura. Los resultados evidencian un incremento del vocabulario semántico y fonológico en contextos conversacionales con una mejoría en el proceso de evocación, aumentando el número de palabras producidas.

En referencia a la dimensión pragmática del lenguaje, Ios pacientes mejoran en el uso de habilidades no verbales, mostrando mayor facilidad en el inicio detópico conversacional e incorporando en su discurso estrategias para realizar demandas de información y recomposición de rupturas comunicativas, aunque la presencia de anomia continua se reduce su incidencia.

\section{DISCUSIÓN Y CONCLUSIONES}

Los 18 estudios analizados manifiestan haber encontrado cambios positivos en la producción léxica de los pacientes como consecuencia de la repetición y exposición intensa y sistemática de los elementos seleccionados.

En el análisis de todas las investigaciones se evidencian cambios significativos en el lenguaje de los pacientes, relacionado con tareas de nominación, evocación y secuenciación, así como en aspectos suprasegmentales y pragmáticos. Las técnicas básicas de intervención ofrecen resultados positivos, sin necesidad de realizar programas que requieran de soportes digitales complejos. Paralelamente, se recomienda realizar una práctica diaria en el hogar que permita seguir entrenando a los pacientes en el uso de diversas habilidades comunicativas.

Los resultados no son permanentes y su perdurabilidad es variable. Por ello, se sugiere realizar seguimientos 
continuos a lo largo del tiempo. La combinación de terapias intensivas con otras más secuenciadas y distribuidas, favorecerán el mantenimiento de los ítems entrenados durante más tiempo.

Asimismo, emplear la práctica repetida de exposición palabra-dibujo permite mantener el nivel de léxico conocido por el paciente. La estimulación conlleva en todos los estudios consultados la repetición del nombre del elemento, seguido de la exposición a su imagen; es decir, la correspondencia imagen-palabra que puede ser estimulada a través de exposición a la forma ortográfica (palabra escrita) (Mayberry et al., 2011) o la forma fonológica (palabra hablada) (Savage et al., 2013).

En este sentido, hay que apuntar que, los beneficios obtenidos al proporcionar claves fonológicas y ortográficas se obtienen en la producción escrita y no en la oral (Savage et al., 2013). Además, para obtener resultados positivos si se trabaja con palabras desconocidas para los pacientes, se deberá implementar un aprendizaje sin error (Senaha et al., 2010).

En referencia al reaprendizaje de vocabulario en personas con demencia, una de las variables relacionadas con el éxito en las intervenciones es que los participantes mantengan intactas las representaciones semánticas y fonológicas de los ítems que se van a presentar (Henry et al., 2008).

A todo lo anterior hay que añadir, que para prolongar los efectos del entrenamiento lingüístico se, tal y como apuntan Savage et al. (2015), es necesario mantener una práctica o entrenamiento constante pero no intensivo tras la implementación del programa, teniendo en cuenta que el beneficio obtenido es mayor cuanto mayor sea el grado de severidad de la demencia. En estos casos, una pequeña mejoría supone una gran oportunidad de mantenimiento (Krueger et al., 2010). Por ello, el mayor o menor grado de éxito de las investigaciones revisadas se centra en la frecuencia y tiempo destinado al reaprendizaje. Aquellas intervenciones realizadas de forma intensiva, que no realizan seguimiento a posteriori evidencian un declive en los resultados obtenidos inmediatamente después de concluir el programa. En cambio, aquellas intervenciones que alargan su entrenamiento a través de sesiones de seguimiento, mantienen los resultados de una forma más duradera incluso 6 meses después del tratamiento.

Concluimos apoyando la idea de la posibilidad de obtener cambios significativos en el lenguaje de los pacientes con demencia, a nivel expresivo, en tareas de nominación, evocación, secuenciación y en aspectos suprasegmentales y pragmáticos cuando se implementan programas de naturaleza cuasiexperimental con una práctica intensiva seguida de un mantenimiento longitudinal mas allá de los 6 meses post-tratamiento, momento en el que las evidencias analizadas reflejan un declive significativo.

\section{LIMITACIONES Y PERSPECTIVAS FUTURAS}

Las limitaciones fundamentales detectadas en el análisis de las investigaciones previas se sintetizan en la escasez de muestra poblacional, la diversidad de cuadros clínicos de los pacientes seleccionados junto a la imposibilidad de poner en práctica los programas en sesiones distanciadas que favorecerían de este modo el entrenamiento cognitivo durante mas tiempo. Se impide la realización de generalizaciones de rango mayor acerca de los beneficios que aporta el desarrollo de programas lingüísticos destinados a pacientes con demencia.

Unido a esto, tal y como se apuntó en líneas anteriores, la gran variabilidad de cuadros clínicos que cursan con deterioro cognitivo obliga a seleccionar pacientes

dispares, tomando criterios simples de inclusión inicial, ya que las distancias entre pacientes durante el desarrollo del programa se harán cada vez más significativas debido a la degeneración progresiva característica del deterioro cognitivo.

En referencia a la metodología utilizada y la temporalización en su implementación, se prueba que una práctica intensiva enmarcada dentro de un periodo breve de tiempo no beneficia al mantenimiento de los logros obtenidos con la implementación del programa. Para que estos se prolonguen en el tiempo, será necesario realizar sesiones de seguimiento a posteriori que refuercen los reaprendizajes.

Nuestra perspectiva de futuro propone integrar las conclusiones de este estudio en el diseño de un programa 
lingüístico destinado a personas con demencia, con la inclusión de actividades que estimulen no solo la recuperación léxica sino los aspectos conversacionales y pragmáticos del lenguaje.

\section{REFERENCIAS BIBLIOGRÁFICAS}

Beales, A., Cartwright, J., Whitworth, A., \& Panegyres, P. K. (2016). Exploring generalisation processes following lexical retrieval intervention in primary progressive aphasia. International Journal of Speech-Language Pathology, 18(3), 299-314. https://doi.org/10.3109/17549507.2016.1151936

Beeson, P. M., King, R. M., Bonakdarpour, B., Henry, M. L., Cho, H., \& Rapcsak, S. Z. (2011). Positive Effects of Language Treatment for the Logopenic Variant of Primary Progressive Aphasia. Journal of Molecular Neuroscience, 45(3), 724-736. https://doi.org/10.1007/s12031-011-9579-2

Bourgeois, M.S. \& Hickey, E.M. (2009). Dementia: From diagnosis to management: A functional approach. Psychology Press.

Brambati, S.M., Rankin, K.P., \& Narvid, J. (2009). Atrophy progression in semantic dementia with asymmetric temporal involvement: A tensor-based morphometry study. Neurobiol Aging, 30, 103-111.

Croot, K., Taylor, C., Abel, S., Jones, K., Krein, L., Hameister, I., Ruggero, L., \& Nickels,

L. (2015). Measuring gains in connected speech following treatment for word retrieval: A study with two participants with primary progressive aphasia. Aphasiology, 29(11), 1265-1288. https://doi.org/10.1080/02687038.2014.975181

García, J.J. \& Carro, J. (2011). Programa de Actuación Cognitiva Integral en Demencias (PACID): Centro de Referencia Estatal de Atención a Personas con Enfermedad de Alzheimer y otras Demencias (1 ${ }^{\mathrm{a}}$ ). Ministerio de Sanidad, Política Social e Igualdad.

Henry, M. L., Beeson, P. M., \& Rapcsak, S. Z. (2008). Treatment for lexical retrieval in progressiveaphasia. Aphasiology, 22(7-8), 826-838. https://doi.org/10.1080/02687030701820055

Henry, M.L., Rising, K., DeMarco, A.T., Miller, B.L., Gorno-Tempini, M.L., \& Beeson,

P.M. (2013). Examining the value of lexical retrieval treatment in primary progressive aphasia: Two positive cases. Brain and Language, 127, 145-156.

Heredia, C. G., Sage, K., Ralph, M. A. L., \& Berthier, M. L. (2009). Relearning and retention of verbal labels in a case of semantic dementia. Aphasiology, 23(2), 192- 209. https://doi.org/10.1080/02687030801942999

Jokel, R., \& Anderson, N. D. (2012). Quest for the best: Effects of errorless and active encoding on word re-learning in semantic dementia. Neuropsychological Rehabilitation, 22(2), 187-214. https://doi.org/10.1080/09602011.2011.639626

Jokel, R., Cupit, J., Rochon, E., \& Leonard, C. (2009). Relearning lost vocabulary in nonfluent progressive aphasia with MossTalk Words $\circledast$. Aphasiology, 23(2), 175- 191. https://doi.org/10.1080/02687030801943005

Jokel, R., Graham, N., Rochon, E., \& Leonard, C. (2014). Word retrieval therapies in primary progressive aphasia. Aphasiology, 28, 1038-1068.

Jokel, R., Rochon, E., \& Anderson, N. D. (2010). Errorless learning of computer- generated words in a patient with semantic dementia. Neuropsychological Rehabilitation, 20(1), 16-41. https://doi.org/10.1080/09602010902879859

Krueger, C.E., Dean, D.L., \& Rosen, H.J. (2010). Longitudinal rates of lobar atrophy in frontotemporal dementia, semantic dementia, and Alzheimer's disease. Alzheimer Dis Assoc Disord. 2010;24: 43-48. 24, 43-48.

Mayberry, E. J., Sage, K., Ehsan, S., \& Lambon Ralph, M. A. (2011). Relearning in semantic dementia reflects contributions from both medial temporal lobe episodic and degraded neocortical semantic systems: Evidence in support of the complementary learning systems theory. Neuropsychologia, 49(13), 3591-3598. https://doi.org/10.1016/j.neuropsychologia.2011.09.010

McKhann, G.M., Knopman, D.S., Chertkow, H., Hyman, B.T., Jack, C.R., Kawas, C.H., \& Phelps, C.H. (2011). The diagnosis of dementia due to Alzheimer's disease: Recommendations from the National Institute on Aging- 
Alzheimer's Association workgroups on diagnostic guidelines for Alzheimer's disease. Alzheimer $s$ \& Dementia: the Journal of the Alzheimer s Association, 7, 263-269.

Mesulam, M. M., Rogalski, E. J., Wieneke, C., Hurley, R. S., Geula, C., Bigio, E. H., Thompson, C. K. \& Weintraub, S. (2014). Primary progressive aphasia and the evolving neurology of the language network. Nature Reviews Neurology, 10(10), 554-569.

Riaño, J. R., \& Moreno, D. M. B. (2012). Beneficios de un programa de estimulación cognoscitivo /comunicativo en adultos con deterioro cognitivo moderado derivado de demencia. Areté, 12, 128-139.

Robinson, S., Druks, J., Hodges, J., \& Garrard, P. (2009). The treatment of object naming, definition, and object use in semantic dementia: The effectiveness of errorless learning. Aphasiology, 23(6), 749-775.

https://doi.org/10.1080/02687030802235195

Savage, S. A., Ballard, K. J., Piguet, 0., \& Hodges, J. R. (2013). Bringing words back to mind - Improving word production in semantic dementia. Cortex, 49(7), 1823- 1832. https://doi.org/10.1016/j.cortex.2012.09.014

Savage, S. A., Piguet, 0., \& Hodges, J. R. (2014). Giving Words New Life: Generalization of Word Retraining Outcomes in Semantic Dementia. Journal of Alzheimer's Disease, 40(2), 309-317. https://doi.org/10.3233/JAD-131826

Savage, S., Piguet, 0., \& Hodges, J. (2015). Cognitive intervention in semantic dementia. Maintaining Words Over Time. Alzheimer Disease and Associated Disorders, 29(1), 55-62.

Senaha, M. L. H., Brucki, S. M. D., \& Nitrini, R. (2010). Rehabilitation in semantic dementia: Study of the effectiveness of lexical reacquisition in three patients. Dementia \& Neuropsychologia, 4, 306-312. https://doi.org/10.1590/S1980- 57642010DN40400009

Suárez-González, A., Heredia, C. G., Savage, S. A., Gil-Néciga, E., García-Casares, N., Franco-Macías, E., Berthier, M. L., \& Caine, D. (2015). Restoration of conceptual knowledge in a case of semantic dementia. Neurocase, 21(3), 309-321.

https://doi.org/10.1080/13554794.2014.892624

Triviño, M., Arnedo, M., \& Bembibre, J. (2021). Neuropsicología a través de Casos Clínicos. Evaluación y rehabilitación (2a ed.). Médica Panamericana. 
